\title{
A influência da informação publicada nas "redes sociais" no processo de seleção e recrutamento: um estudo exploratório na literatura
}

\section{The influence of the information published in "social networks" in the process of selection and recruitment: an exploratory study in the literature}

\author{
Luís Miguel Oliveira Machado ${ }^{1}$ \\ ${ }^{1}$ Universidade de Coimbra - UC, Coimbra, Portugal
}

Autor para correspondência/Mail to: Luís Miguel Oliveira Machado (luismmachado70@gmail.com)

Recebido/Submitted: 23 Nov. 2015; Aceito/Approved: 14 Fev. 2016

Copyright (C) 2016 Machado, L. M. O.. Todo o conteúdo da Revista (incluindo-se instruções, política editorial e modelos) está sob uma licença Creative Commons Atribuição-NãoComercial-Compartilhalgual 3.0 Não Adaptada. Ao serem publicados por esta Revista, os artigos são de livre uso em ambientes educacionais, de pesquisa e não comerciais, com atribuição de autoria obrigatória. Mais informações em http://ojs.c3sl.ufpr.br/ojs2/index.php/atoz/about/submissions\#copyrightNotice.

\begin{abstract}
Resumo
Introdução: Numa sociedade cada vez mais online a relação entre pessoas alastrou-se naturalmente para a rede, para sites conhecidos como "Redes Sociais". Nessa replicação, certos modos de comunicar e se representar, inócuos em estado offline, podem ser bastante prejudiciais profissionalmente quando reproduzidos em linha. Assim, o objetivo desta investigação é refletir sobre a importância - em termos profissionais do perfil que é criado nas, ou pelo uso das, Redes Sociais em Linha (como o Facebook e outros sites similares), considerando a influência da atividade aí desenvolvida na obtenção de uma contratação laboral.

Método: Foi realizada uma revisão da literatura, em forma de pesquisa exploratória, incluindo fontes bibliográficas e documentais, entre as quais 16 estudos primários efetuados entre 2008 e 2014, abrangendo a perspetiva de investigadores e recrutadores sobre esta problemática de forma a responder três questões, a saber: a) Os responsáveis pelos recursos humanos e/ou outros com poder de decisão em termos de gestão de funcionários, procuram efetivamente informação em linha sobre potenciais funcionários? b) A informação encontrada tem um papel relevante na decisão desses gestores de recursos humanos? c) Quem procura trabalho deve cuidar da sua "imagem digital" mesmo nas Redes Sociais em Linha não consideradas profissionais?

Resultados: A procura de informação nas chamadas "Redes Sociais" sobre os candidatos a ofertas de emprego é efetivamente realizada ao longo de todo o processo de seleção e recrutamento. Essa prática, denominada de cybervetting, também abrange as Redes Sociais em Linha tidas como não profissionais e, atendendo aos estudos analisados, é de esperar um aumento da sua frequência. A informação encontrada nas "Redes Sociais em Linha" é também considerada na avaliação dos candidatos às ofertas de emprego, uma vez que é entendida, por muitos recrutadores, como parte da "identidade digital" do indivíduo em questão. Verifica-se, igualmente, que a influência da informação obtida por essa via tende a não ser favorável aos candidatos.

Conclusão: Do ponto de vista dos recrutadores a prática de cybervetting é, frequentemente, encarada como um dever profissional no sentido de encontrar o candidato mais adequado. Pelo lado dos candidatos urge uma maior consciencialização da repercussão que a atividade desenvolvida em linha pode ter em termos profissionais. Os efeitos na sua "imagem digital" são similares aos de uma tatuagem, deixa marcas dificilmente removíveis.
\end{abstract}

Palavras-chave: Redes sociais; Oferta de emprego; Recrutamento e seleção; Identidade digital; Cybervetting

\begin{abstract}
Introduction: In a society that goes increasingly online, the relationship between people spread naturally to the network, to sites known as "social networks". In this replication, certain ways of communicating and self-representation, benign in offline state, can be very harmful professionally when played online. Thus, the objective of this research is to reflect on the importance - in professional terms - of the profile that is created on, or by the use of, online social networks (as Facebook and other similar sites), considering the influence of the activity developed there in the attainment of a labor contract. Method: In order to reflect on the influence of the activity performed online - on Facebook or similar sites - in obtaining an employment contract, a literature review was conducted in the form of exploratory research. It included bibliographical and documentary sources, among which 16 primary studies conducted between 2008 and 2014, covering the perspective of researchers and recruiters on this issue seeking to answer three questions, as follows: a) those responsible for human resources and/or other decision-makers, regarding employee management, effectively seek online information about potential employees? b) the information found has a relevant role in the decision of these HR managers? c) those, who are looking for a job, should take care of their "digital image" even in Social Networks considered nonprofessional?

Results: The request for information, in the so-called "Social Networks", is performed along the process of selection and recruitment of candidates for job vacancies. This practice, called cybervetting, also covers online social networks taken as non-professional and, taking into account the studies analyzed, is expected an increase of its frequency. The information found there is also considered in the assessment of the candidates of job vacancies since as it is understood, by many recruiters, as part of the "digital identity" of the individual in question. It is verified, equally, that the influence of the information obtained in this way tends not to be favorable to the potential employee.

Conclusions: From the point of view of recruiters, the practice of cybervetting is often seen as a professional duty to find the most suitable candidate. On the side of the candidates urges greater awareness of the impact that online activity may have professionally. The effects on their "digital image" are similar to those of a tattoo, leaves hardly removable marks.
\end{abstract}

Keywords: Social Networks; Job vacancies; Recruitment and selection; Digital identity; Cybervetting 


\section{INTRODUÇÃO}

A Word Wide Web, ou simplesmente a Web, tem vindo a sofrer uma mudança gradual na finalidade para a qual é maioritariamente utilizada sendo a utilização enquanto meio de comunicação o motor dessa mudança. Uma transformação da "sociedade da informação" para uma "sociedade da comunicação" parece estar em curso (Cardoso, Araújo, \& Espanha, 2009, p. 3). Uma comunicação sempre presente, independente do lugar ou tempo, graças à proliferação dos dispositivos móveis, que permitem o acesso à Web, e à facilidade (operacional e económica) desse acesso, dando origem à denominada geração “always on" (Cardoso et al., 2009, p. 4).

Nesta "sociedade da comunicação", sites como Facebook, Google+, Hi5, entre outros, são uma continuação natural das Redes Sociais, criadas pelo complexo sistema de relacionamentos interpessoais, essenciais às comunidades humanas desde tempos ancestrais (Araújo, Cardoso, e Espanha, 2008, p. 4; Harvard Medical School, 2012, 3:23).

No presente trabalho será usada a designação "Redes Sociais em Linha” (RSeLs), englobando o conjunto de pessoas empaticamente ligadas (a "rede social" propriamente dita) e a aplicação informática que, utilizando a internet, serve de canal de comunicação e "local de reunião" desse conjunto de pessoas. A opção por esta designação decorre da ênfase do trabalho ser colocada na "atividade social realizada em linha" (Ellison \& Boyd, 2013, p. 158), e de não existir em Portugal um termo de uso tão consensual, como "redes sociais virtuais" utilizado no Brasil, que precise o conceito.

Como parte da atividade desenvolvida nas Redes Sociais em Linha, comentários e/ou imagens, os chamados posts, colocadas em áreas encaradas como privadas por quem as coloca, têm sido a causa de despedimento dos seus autores, por os mesmos serem considerados ofensivos pelas entidades empregadoras (Campos \& Oliveira, 2013). Mesmo em terras lusas alguns casos têm sido levados a tribunal com diferentes desfechos (Saramago, 2015; SIC Notícias, 2015).

Estes casos serão o lado visível da questão, uma vez que a consequência, o despedimento, é perfeitamente identificado com a causa, os comentários efetuados em linha. Já no caso da não obtenção de uma contratação laboral devido a essa mesma causa será bastante mais difícil, ou quiçá impossível, de relacionar. Mesmo a recente diretiva do Conselho da Europa sobre o tratamento de dados pessoais no contexto do emprego (Council of Europe, 2015) de pouco servirá ao alegado lesado, não sendo possível estabelecer a tal relação.

Neste contexto, saber qual a relevância dada, pelos potenciais empregadores, à "atividade social" realizada em linha, é de particular interesse a todos que pretendam uma mudança, e até mesmo a manutenção, da sua situação profissional.

\section{OBJETIVOS E METODOLOGIA}

Com este trabalho pretende-se, através de uma revisão da literatura, refletir sobre a importância em termos profissionais do perfil que é criado nas, ou pelo uso das Redes Sociais em Linha.

Tendo em conta o objetivo enunciado, foram selecionadas três questões para a sua consecução:

a) Os responsáveis pelos recursos humanos e/ou outros com poder de decisão em termos de gestão de funcionários, procuram efetivamente informação em linha sobre potenciais funcionários?

b) A informação encontrada tem um papel relevante na decisão desses gestores de recursos humanos?

c) Quem procura trabalho deve cuidar da sua "imagem digital” mesmo nas RSeLs não consideradas profissionais?

Metodologicamente, este trabalho assume-se como uma pesquisa exploratória uma vez que que se procurou abranger uma grande variedade de perspetivas sobre a problemática em questão. Assim, recorreu-se tanto a fontes bibliográficas como documentais de primeira e segunda mão (Gil, 2008, p. 51), buscando diversidade no contexto e responsabilidade da sua produção.

Relativamente aos estudos primários consultados (Cf. Tabela 1 e Quadro 1), para além da diversidade de perspetivas em termos das entidades que os realizam, pretendeu-se que os mesmos fossem recentes, definindose uma janela temporal entre 2008 e 2014 e, apesar de não limitar a área geográfica da sua incidência, procurou-se - em particular - trabalhos produzidos em Portugal e no Brasil.

A opção pela abrangência de perspetivas justifica o alargamento da área geográfica dado o número reduzido de estudos, realizados em Portugal e no Brasil, encontrados. No que concerne a produções científicas desses países foi utilizado o Portal Brasileiro de Publicações Científicas em Acesso Aberto (OASISbr), uma vez que o mesmo inclui o seu congênere português, o Repositório Científico de Acesso Aberto de Portugal (RCAAP), como fonte e a sua plataforma possibilita uma pesquisa rápida. Pesquisou-se no campo "Título" e no campo "Assunto" utilizando o termo de pesquisa "redes sociais" em combinação, utilizando o operador booleano "AND”, 


\begin{tabular}{|c|c|c|c|c|}
\hline Ref. & Datas $^{1}$ & Título do documento & País(es) de incidência & Dimensão da amostra ${ }^{2}$ \\
\hline$A a^{3}$ & 2008 jul.-set. & $\begin{array}{l}\text { Online technologies and their impact on re- } \\
\text { cruitment strategies: Using social networking } \\
\text { websites to attract talent. }\end{array}$ & Estados Unidos ${ }^{4}$. & 571 \\
\hline$A b^{3}$ & & $\begin{array}{l}\text { Staffing research: Online technologies and } \\
\text { their impact on recruitment strategies. }\end{array}$ & & \\
\hline B & 2009 dez.10-23 & Online reputation in a connected world. & $\begin{array}{l}\text { Alemanha; França; Esta- } \\
\text { dos Unidos; Reino Unido. }\end{array}$ & $1106^{5}$ \\
\hline C & 2010 mai.-jun. & 2010: Social recruiting survey results. & Estados Unidos. & 600 \\
\hline $\mathrm{D}$ & 2010 dez.17-2011 fev.1 & $\begin{array}{l}\text { SHRM Survey Findings: The use of social } \\
\text { networking websites and online search en- } \\
\text { gines in screening job candidates. }\end{array}$ & Estados Unidos ${ }^{4}$. & 541 \\
\hline$E$ & 2011 & Recrutamento nas redes sociais on-line. & Portugal. & 13 \\
\hline $\mathrm{F}$ & 2011 & $\begin{array}{l}\text { Redes sociais virtuais como uma nova fer- } \\
\text { ramenta nos processos de recrutamento, } \\
\text { seleção e controle de pessoal. }\end{array}$ & Brasil. & 5 \\
\hline G & 2012 & $\begin{array}{l}\text { A influência das redes sociais virtuais no } \\
\text { processo de recrutamento e seleção das } \\
\text { empresas associadas à AJESM. }\end{array}$ & Brasil. & 29 \\
\hline $\mathrm{H}$ & 2012 & $\begin{array}{l}\text { Uso das redes sociais virtuais no processo } \\
\text { de recrutamento e seleção de pessoal: } \\
\text { Uma análise na perspectiva de profissio- } \\
\text { nais de recursos humanos. }\end{array}$ & Brasil. & 8 \\
\hline 1 & 2012 & $\begin{array}{l}\text { Impacto das novas tecnologias no recru- } \\
\text { tamento nas empresas especializadas de } \\
\text { recrutamento e selecção. }\end{array}$ & Portugal. & 58 \\
\hline$J$ & 2012 out. $20-24$ & $\begin{array}{l}\text { O uso das redes sociais virtuais nos pro- } \\
\text { cessos de recrutamento e seleção. }\end{array}$ & Brasil. & 15 \\
\hline $\mathrm{K}$ & 2013 mar.7-14 & $\begin{array}{l}\text { The use of social media in the recruitment } \\
\text { process. }\end{array}$ & Reino Unido. & 401 \\
\hline M & 2014 abr.14-mai.29 & $\begin{array}{l}\text { O uso das redes sociais no recrutamento } \\
\text { externo em empresas portuguesas. }\end{array}$ & Portugal. & 5 \\
\hline $\mathrm{N}$ & 2014 mar.1-jul.28. & $\begin{array}{l}\text { A procura online dos talentos: O papel dos } \\
\text { sites de redes sociais no recrutamento de } \\
\text { candidatos }\end{array}$ & Portugal. & 15 \\
\hline $\mathrm{O}$ & 2014 ago & 2014: Social recruiting survey. & Estados Unidos. & 1855 \\
\hline $\mathrm{Pa}^{6}$ & 2014 & $\begin{array}{l}\text { Cybervetting, person-environment fit, and } \\
\text { personnel selection: Employers' surveillance } \\
\text { and sensemaking of job applicants' online in- } \\
\text { formation. }\end{array}$ & Estados Unidos. & 45 \\
\hline $\mathrm{Pb}^{6}$ & & $\begin{array}{l}\text { Online employment screening and digital ca- } \\
\text { reer capital: Exploring employers' use of on- } \\
\text { line information for personnel selection. }\end{array}$ & Estados Unidos. & \\
\hline
\end{tabular}

Tabela 1. Estudos primários consultados para o presente trabalho.

Fonte: elaborado pelo autor.

Notas: (1) As datas apresentadas dizem respeito ao período da recolha de dados. Nos casos em que não foi possível obter essa informação, a data apresentada é a da publicação do estudo. (2) Profissionais com poder de decisão em termos de recrutamento e seleção de funcionários. (3) Os documentos referenciados como Aa e Ab abordam aspetos diferentes do mesmo estudo. (4) O estudo contou com a participação de profissionais de outros países não especificados para além dos Estados Unidos que contribuiu com cerca de $75 \%$ da amostra. (5) A distribuição da amostra pelos países participantes foi a seguinte: 275 - Estados Unidos; 276 - Reino Unido; 279 - Alemanha; 275 - França. (6) Os documentos referenciados como $\mathrm{Pa}$ e $\mathrm{Pb}$ são artigos que aprofundam aspetos diferentes do mesmo estudo.

alternadamente com os termos "emprego", "recrutamento" e "seleção", aplicando a truncatura aos mesmos ("empreg*”, "recruta*”, "selec*") de forma a recuperar as suas variações.

Para além dos estudos incluídos na Tabela 1, considerou-se pertinente utilizar dados obtidos de forma indireta em dois casos distintos. No caso do estudo efetuado pela entidade Social Media Monitoring Service Reppler, embora não tenha sido possível obter o documento, os dados do mesmo estão disponíveis de forma consistente em várias fontes. No segundo caso, considerou-se pertinente a utilização dos dados do artigo "Employer’s use of social networking sites” (Clark \& Roberts, 2010), na Tabela 7, pela credibilidade da fonte ${ }^{1}$.

\footnotetext{
${ }^{1}$ Artigo publicado no Journal of Business Ethics, Editora Springer.
} 


\begin{tabular}{|c|c|c|c|}
\hline \multicolumn{3}{|c|}{ Contextualização } & \multirow[t]{2}{*}{ Estudos (Ref. Tabela 1) } \\
\hline \multicolumn{2}{|l|}{ Entidade } & Tipo de documento & \\
\hline \multirow{3}{*}{\multicolumn{2}{|c|}{ Instituição de Ensino Superior: }} & Artigo científico & $\mathrm{G}, \mathrm{H}, \mathrm{J}, \mathrm{P}$ \\
\hline & & Dissertação de mestrado $^{1}$ & $\mathrm{E}, \mathrm{I}, \mathrm{M}, \mathrm{N}$ \\
\hline & & Trabalho final de bacharelato $^{2}$ & $\mathrm{~F}$ \\
\hline \multirow{3}{*}{ Organização privada } & \multirow{2}{*}{ ligada aos recursos humanos } & Whitepaper & $\mathrm{L}$ \\
\hline & & \multirow{3}{*}{ Relatório de estudo } & $A^{3}, C, D^{3}, O$ \\
\hline & de estudos de mercado & & $\mathrm{B}^{4}$ \\
\hline \multicolumn{2}{|c|}{$\begin{array}{l}\text { Organismo internacional independente de pes- } \\
\text { quisa e consultadoria relacionadas com emprego: }\end{array}$} & & $\mathrm{K}^{5}$ \\
\hline
\end{tabular}

Quadro 1. Contextualização dos estudos primários consultados para o presente trabalho.

Fonte: elaborado pelo autor.

Notas: (1) Em Gestão e/ou Desenvolvimento de Recursos Humanos. (2) Em Administração. (3) Estudos realizados pela Society for Human Resource Management (SHRM), aos seus afiliados. (4) Estudo encomendado pela Microsoft. (5) Estudo encomendado pela organização não-governamental Advisory, Conciliation and Arbitration Service (ACAS).

\section{A PROCURA DE INFORMAÇÃO EM LINHA SOBRE OS CANDIDATOS}

De acordo com todos os estudos consultados, variando apenas em termos percentuais, a resposta à primeira questão é afirmativa no contexto do universo de literatura estudado: os responsáveis pelos recursos humanos, e/ou outros com poder de decisão em termos de gestão de funcionários, procuram efetivamente informação em linha sobre os candidatos às ofertas de emprego.

Não sendo possível fazer comparações em termos de expressividade das percentagens, dada a disparidade dos vários estudos quer no tempo (2008 a 2014), quer no país envolvido, quer na dimensão amostral, alguns dados, porém, poderão ser confrontados como material de reflexão. Por exemplo, dois estudos efetuados entre maio de 2010 e janeiro de 2011 nos Estados Unidos, apontam para resultados muito distintos a questões similares, como se pode ver na Tabela 2.

Do mesmo modo, resultados próximos também foram encontrados. Como exemplo, pode-se referir dois estudos realizados no Reino Unido entre março e julho de 2013 Tabela 3.

\begin{tabular}{lllc}
\hline Estudo & Questão colocada & Resposta selecionada & Percentagem contabilizada \\
\hline C & How do you use online profiles when revi- & Do not review profiles at all. & $16,2 \%$ \\
D & $\begin{array}{l}\text { ewing candidates? } \\
\text { Has your organization used social networ- } \\
\text { king websites to screen job candidates at any } \\
\text { point in the hiring process, or does it plan to } \\
\text { do so? }\end{array}$ & $\begin{array}{l}\text { Not we have never used this method and do } \\
\text { noto so. }\end{array}$ & $67 \%$ \\
\hline
\end{tabular}

Tabela 2. Exemplo da disparidade de resultados encontrados em estudos a questões similares.

Fonte: elaborado pelo autor.

Notas: O estudo "C" foi efetuado pela Jobvite, uma empresa fornecedora de serviços (B to B) ligados aos recursos humanos (Jobvite, 2010, p. 7) e o "D" pela SHRM (Society for Human Resource Management [SHRM], 2011, p. 6).

\begin{tabular}{lllc}
\hline Estudo & Questão colocada & Resposta selecionada & Percentagem contabilizada \\
\hline $\mathrm{K}$ & $\begin{array}{l}\text { Which of the following best describes your } \\
\text { own organisation's position on using social }\end{array}$ & We never use social media when recruiting & $38,4 \%$ \\
& $\begin{array}{l}\text { media when recruiting staff? } \\
\text { If you view professional social networking } \\
\text { profiles during the recruitment process, at } \\
\text { what stage do you do so? }\end{array}$ & $\begin{array}{l}\text { Don't use professional social networking sites } \\
\text { for this purpose. }\end{array}$ & $33,9 \%$ \\
& & & \\
\hline
\end{tabular}

Tabela 3. Exemplo da proximidade de resultados encontrados em estudos a questões similares.

Fonte: elaborado pelo autor.

Notas: O estudo "K" foi efetuado pelo Institute for Employment Studies (Broughton, Foley, Ledermaier, \& Cox, 2013, p. 56) e o "L" pela empresa de recursos humanos Robert Walter Group (Robert Walter Group, 2013, p. 11).

Num estudo comparativo realizado em 2009 (Tabela 4), as percentagens apuradas são superiores em, aproximadamente, dez pontos percentuais comparando, respetivamente, para os Estados Unidos, com a maior percentagem apresentada na Tabela 2, e para o Reino Unido, com a maior percentagem apresentada na Tabela 3. O referido estudo faz uma confrontação entre a percentagem de companhias que requerem uma verificação da "reputação em linha" dos candidatos e a percentagem de profissionais que a fazem (Cross-Tab, 2010, p. 6).

Dos quatro países, o caso da Alemanha destaca-se com uma diferença de trinta e oito pontos percentuais entre a verificação requerida pelas empresas e a realizada pelos profissionais de recursos humanos, como é visível na Tabela 4. 


\begin{tabular}{lcc}
\hline País & Recrutadores & Empresa \\
\hline Alemanha & $59 \%$ & $21 \%$ \\
Estados Unidos & $79 \%$ & $75 \%$ \\
França & $23 \%$ & $21 \%$ \\
Reino Unido & $47 \%$ & $48 \%$ \\
\hline
\end{tabular}

Tabela 4. Estudo comparativo: "Percent of companies with policies that require review of reputational data vs. percent of recruiters and HR professionals surveyed who seek it".

Fonte: Cross-Tab (2010, p. 6).

Notas: Estudo efetuado pela organização profissional Cross-Tab (estudo com a referencia “B” na Tabela 1).

\section{CYBERVETTING - FERRAMENTA DE SELEÇÃO OU DE "INVASÃO"?}

As percentagens apuradas, embora sejam indicadores consistentes no sentido de perceber a relevância das verificações da atividade realizada em linha pelos candidatos, ou potenciais candidatos, a ofertas de emprego, poderão não revelar toda a extensão do fenómeno. Num estudo qualitativo (referencia "P" da Tabela 1) as autoras concluem que, embora alguns recrutadores neguem essa verificação descrevem comportamentos durante o processo de recrutamento associados à mesma, como por exemplo “doing a quick Google check” (Berkelaar \& Buzzanell, 2015, p. 3).

Esse processo de verificação de informação em linha, denominado de cybervetting na literatura consultada, é realizado nas várias fases do processo de seleção e recrutamento, seja logo após da receção das candidaturas e/ou currículos como forma de selecionar e reduzir o número de candidatos, seja após a entrevista de modo a confirmar ou obter mais informações relativas aos candidatos (Almeri, Martins, Paula, e Paiva, 2013, p. 87; Broughton et al., 2013, p. 60; Jobvite, 2014, p. 8; Molina, 2014, p. 77; Robert Walter Group, 2013, p. 11; Society for Human Resource Management [SHRM], 2008b, p. 7; SHRM, 2011, p. 15).

O processo de cybervetting estende-se a todo o tipo de RSeLs, desde as consideradas profissionais, como o LinkedIn, até às tidas como pessoais como Facebook ou Twitter (ver Tabela 5).

\begin{tabular}{lcccccc}
\hline Estudo & Linkedln & Facebook & Twitter & Google+ & YouTube & Outros \\
\hline $\mathrm{D}$ & $85 \%$ & $78 \%$ & $11 \%$ & - & - & $35 \%$ \\
$\mathrm{I}$ & $78 \%$ & $67 \%$ & $31 \%$ & - & - & $20 \%$ \\
$\mathrm{~J}$ & $60 \%$ & $27 \%$ & $13 \%$ & - & - & - \\
$\mathrm{K}$ & $71 \%$ & $52 \%$ & $31 \%$ & $15 \%$ & - & $34 \%$ \\
$\mathrm{~N}$ & $80 \%$ & $26 \%$ & - & - & - & - \\
$\mathrm{O}$ & $94 \%$ & $66 \%$ & $52 \%$ & $21 \%$ & $15 \%$ & $20 \%$ \\
SMSR1 $^{1}$ & $48 \%$ & $76 \%$ & $53 \%$ & - & - & - \\
\hline
\end{tabular}

Tabela 5. Distribuição, dentro das RSeLs utilizadas para verificação de informação de candidatos, da percentagem da amostra estudada que reportou fazer esse tipo de verificação.

Fonte: elaborado pelo autor.

Notas: Nos "outros", para além de outras RSeLs como: Hi5; theStarTracker; Orkut; FormSpring.me; MySpace, em alguns estudos também é incluído motores de busca e blogs pessoais. (1) As percentagens do estudo da entidade Social Media Monitoring Service Reppler foram obtidas de forma indireta (Macleod, 2011).

A abundância e facilidade de obtenção de informação por esta via (Cross-Tab, 2010, p. 20; L’Atelier, 2009; Openshaw, 2011, p. 8) é encarada por muitos recrutadores como uma mais-valia para a escolha dos candidatos uma vez que lhes possibilita uma visão mais detalhada, dando acesso à "pessoa real” (Almeri et al., 2013, p. 84; Berkelaar e Buzzanell, 2014, p. 269, 2015, p. 99; Fernandes, 2014, p. 25; A. F. P. Gomes, 2011, p. 67; Openshaw, 2011, p. 14). Como tal, é considerada uma ferramenta que deve ser otimizada (Archana, Nivya, e Thankam, 2008, p. 40; Jobvite, 2014, p. 2; Real, Cardoso, Giovaneta, e Birrer, 2012, p. 13).

Em contrapartida, outros autores veem, nessa mesma abundância e facilidade de obtenção de informação, razões de preocupação. Em primeiro lugar, a facilidade de obtenção de informação levanta questões relacionadas com a privacidade dos candidatos (Cross-Tab, 2010, p. 20; Davison, Maraist, Hamilton, e Bing, 2012, p. 18; A. F. P. Gomes, 2011, p. 57). Já a abundância da informação traz problemas de filtragem, dificultando a seleção de informação relevante para o processo de recrutamento (L'Atelier, 2009; Robert Walter Group, 2013, p. 14; SHRM, 2011, p. 2). Por fim, a opinião expressa vai no sentido contrário à da "visão da pessoa real”, contrapondo que a informação recolhida apenas mostra uma parte do todo e pode ser bastante incorreta ou mal interpretada (Berkelaar e Buzzanell, 2014, p. 472; Clark e Roberts, 2010, p. 510; Robert Walter Group, 2013, p. 14).

A relação entre a facilidade na obtenção de informação mais detalhada e a possibilidade de invasão de privacidade, poderá estar na origem de uma certa incongruência entre alguns dados apurados nos estudos, como no caso já mencionado do estudo "P" da Tabela 1 ou o estudo "L" da mesma Tabela (Tabela 6).

A discrepância percentual de mais de trinta pontos, como se pode ver na Tabela 6, entre as duas respostas parece apontar nesse mesmo sentido de dualidade de atitudes. 


\begin{tabular}{lll}
\hline Questão colocada & Resposta selecionada & Percentagem contabilizada \\
\hline $\begin{array}{l}\text { Do you agree that Facebook and Twitter should be } \\
\text { regarded as personal and not professional? }\end{array}$ & Yes. & \\
$\begin{array}{ll}\text { Which of the following viewpoints describes your ap- } \\
\text { proach to researching prospective candidates on per- } \\
\text { sonal social media (eg Facebook and Twitter)? }\end{array}$ & $\begin{array}{l}\text { We do not and would not check social networking sites } \\
\text { during the recruitment process. }\end{array}$ & \\
\hline
\end{tabular}

Tabela 6. Discrepância percentual, no mesmo estudo, em duas respostas relacionadas entre si.

Fonte: elaborado pelo autor.

Notas: Dados recolhidos dos gráficos 2.0 (p. 2) e 6.0 (p. 9) do estudo realizado pela empresa de recursos humanos Robert Walter Group (Robert Walter Group, 2013).

\section{RELEVÂNCIA DA INFORMAÇÃO ENCONTRADA PARA O PROCESSO DE RECRUTAMENTO}

Relativamente às decisões decorrentes da informação encontrada em linha pelos recrutadores, os documentos consultados apresentam mais dados relativos a decisões prejudiciais que favoráveis para os candidatos. A Tabela 7 apresenta uma sistematização desses dados.

\begin{tabular}{|c|c|c|c|c|c|c|}
\hline \multicolumn{2}{|c|}{ Estudo } & \multicolumn{2}{|r|}{ Amostra sobre a qual recai a decisão } & \multicolumn{3}{|c|}{ Decisão reportada } \\
\hline Ref. & Ano & $\%$ & Descrição & $\%$ & Descrição & Tipo $^{1}$ \\
\hline \multirow[t]{2}{*}{1} & 2006 & $25 \%$ & $\begin{array}{l}\text { of hiring managers have conducted Internet } \\
\text { searches (i.e., Google). }\end{array}$ & - & [não é referido] & - \\
\hline & & $12 \%$ & $\begin{array}{l}\text { of employers report searching SNSs for infor- } \\
\text { mation on employers. }\end{array}$ & $63 \%$ & $\begin{array}{l}\text { said they did not hire because of information } \\
\text { found. }\end{array}$ & Neg. \\
\hline \multirow[t]{2}{*}{2} & 2007 & $50 \%$ & $\begin{array}{l}\text { of human-resources professionals ran an In- } \\
\text { ternet search (Google, Yahoo!). }\end{array}$ & $20 \%$ & $\begin{array}{l}\text { who ran searches said they have disqualified } \\
\text { a candidate based on what they found. }\end{array}$ & Neg. \\
\hline & & $15 \%$ & $\begin{array}{l}\text { of human-resources professionals reported } \\
\text { checking SNSs. }\end{array}$ & & & \\
\hline 3 & 2008 & $44 \%$ & $\begin{array}{l}\text { employers reported checking SNSs for infor- } \\
\text { mation. }\end{array}$ & $82 \%$ & $\begin{array}{l}\text { reported that they would let something nega- } \\
\text { tive on the SNSs impact their hiring decision }\end{array}$ & Neg. \\
\hline 4 & 2008 & $21 \%$ & $\begin{array}{l}\text { of employers reported checking SNSs for in- } \\
\text { formation. }\end{array}$ & $34 \%$ & $\begin{array}{l}\text { reported finding content which caused them } \\
\text { to dismiss the person from consideration. }\end{array}$ & Neg. \\
\hline \multirow[t]{2}{*}{ A } & 2008 & $44 \%$ & $\begin{array}{l}\text { HR professionals indicated that they had } \\
\text { used social networking sites as a tool in re- }\end{array}$ & $49 \%$ & $\begin{array}{l}\text { (consequently they) would be mush or so- } \\
\text { mewhat less likely to hire. }\end{array}$ & Neg. \\
\hline & & & & $20 \%$ & $\begin{array}{l}\text { (consequently they) would be mush or so- } \\
\text { mewhat more likely to hire. }\end{array}$ & Pos. \\
\hline 5 & 2009 & $25 \%$ & $\begin{array}{l}\text { employers reported they had looked at } \\
\text { SNSs/online for information. }\end{array}$ & $52 \%$ & $\begin{array}{l}\text { said the information impacted hiring decisi- } \\
\text { ons. }\end{array}$ & Ind. \\
\hline \multirow[t]{2}{*}{ D } & 2010 & $18 \%$ & $\begin{array}{l}\text { organizations that have used social networ- } \\
\text { king websites to screen job candidates. }\end{array}$ & $17 \%$ & $\begin{array}{l}\text { organization that disqualified a job candidate } \\
\text { because of information found (in the past } 12 \\
\text { months). }\end{array}$ & Neg. \\
\hline & & $26 \%$ & $\begin{array}{l}\text { organizations that have used online search } \\
\text { engines to screen job candidates. }\end{array}$ & & & \\
\hline \multirow[t]{2}{*}{ SMSR } & 2011 & $91 \%$ & $\begin{array}{l}\text { employers use social media channels such as } \\
\text { Twitter, Facebook and Linkedln to screen job } \\
\text { applicants. }\end{array}$ & $69 \%$ & $\begin{array}{l}\text { (ever) rejected a candidate because of what } \\
\text { you saw about them on a social networking } \\
\text { site. }\end{array}$ & Neg. \\
\hline & & & & $68 \%$ & $\begin{array}{l}\text { (ever) hired a candidate because of what you } \\
\text { saw about them on a social networking site. }\end{array}$ & Pos. \\
\hline G & 2012 & $52 \%$ & $\begin{array}{l}\text { empresas que durante o processo seletivo } \\
\text { costumam ver o perfil dos candidatos. }\end{array}$ & $55 \%$ & $\begin{array}{l}\text { (empresas) sentem-se muito ou em partes } \\
\text { influenciadas pelo conteúdo das redes so- } \\
\text { ciais. }\end{array}$ & Ind. \\
\hline $\mathrm{N}$ & 2014 & $80 \%$ & $\begin{array}{l}\text { entrevistados reconhecem que usam os } \\
\text { sites de redes sociais para realizarem os } \\
\text { seus processos de recrutamento dos ta- } \\
\text { lentos. }\end{array}$ & $100 \%$ & $\begin{array}{l}\text { concordaram que a informação disponível } \\
\text { nos sites de redes sociais afeta a imagem } \\
\text { do candidato. }\end{array}$ & Ind. \\
\hline $\mathrm{O}$ & 2014 & $55 \%$ & $\begin{array}{l}\text { recruiters have reconsidered a candidate ba- } \\
\text { sed on their social profile. }\end{array}$ & $61 \%$ & (of them are) negative reconsiderations. & Neg. \\
\hline
\end{tabular}

Tabela 7. Decisões decorrentes da informação encontrada em linha pelos recrutadores.

Fonte: elaborado pelo autor.

Notas: Os dados apresentados referentes aos estudos com referência numérica constam no artigo Employer's Use of Social Networking Sites: $A$ Socially Irresponsible Practice (Clark \& Roberts, 2010, p. 510). (1) Na coluna "Tipo" as decisões foram tipificadas em três categorias: decisões negativas para os candidatos - "Neg."; decisões positivas para os candidatos - "Pos." e decisões indiferenciadas - "Ind.". (2) Estudo da entidade Social Media Monitoring Service Reppler (Macleod, 2011). 
Alguns profissionais de recursos humanos também alertam para a influência tendencialmente negativa da informação encontrada nas RSeLs (Martins, 2014, p. 26; Society for Human Resource Management [SHRM], 2008a, p. 5). Referindo-se a essa fonte de informação, Nuno Troni, gestor executivo em Portugal da empresa de recrutamento Michael Page, afirma "Dificilmente beneficia, facilmente prejudica"(Pereira, 2013). Uma opinião que resume as preocupações já aqui referidas, relativas à relevância e descontextualização da informação encontrada. Preocupações que, segundo (Berkelaar \& Buzzanell, 2015, p. 96), parecem ter fundamento:

When making sense of online artifacts, employers consistently recontextualized information through an employment lens. Thus, even if a job candidate's behavior might be considered appropriate for a picture's original context or intended audience, employers disqualified candidates whose online information violated norms for employment audiences or contexts. "I wouldn't hire them", participants repeatedly.

A questão aparenta ser bastante relevante pois, como afirma Alice Weightman, diretora da agência de recrutamento Hanson Search, "People do use social networks to do background checks and, once you've seen something, it's very hard to wipe an image out of your mind" (Bashford, 2008).

\section{"IDENTIDADE DIGITAL": O CARTÃO-DE-VISITA VIRTUAL}

Para Tom Krieglstein, student launcher fundador do projeto Swift Kick, a "identidade digital", criada pela associação dos conteúdos colocados em linha à pessoa que os coloca, chega mesmo a ser considerada mais importante que a "identidade física" em termos profissionais, uma vez que a "primeira impressão" que os empregadores têm dos candidatos é cada vez mais proporcionada pela informação colhida em linha (WETM-TV, 2014, 0:44). Dados recolhidos num estudo realizado nos Estados Unidos assim parecem confirmar:

We created Facebook profiles, manipulating traits, then we started sending out résumés to companies in the U.S., and we detected, we monitored, whether they were searching for our candidates, and whether they were acting on the information they found on social media. And they were. Discrimination was happening through social media for equally skilled candidates (Acquisti, 2013, 7:00).

Advertências sobre o cuidado a ter com a "identidade digital" são bastante recorrentes (Berkelaar e Buzzanell, 2015, p. 108; A. F. P. Gomes, 2011, p. 58; T. C. Gomes, Scherer, e Lobler, 2012, p. 15; Openshaw, 2011, p. 14; SHRM, 2008b, p. 7; WETM-TV, 2014, p. 0:54). Atendendo ao estudo comparativo realizado pela (Cross-Tab, 2010, p. 5) esses avisos são de particular pertinência para os utilizadores das RSeLs dos Estados Unidos e do Reino Unido (Cf. Tabela 8).

\begin{tabular}{lcc}
\hline País & Recrutadores & Consumidores \\
\hline Alemanha & $16 \%$ & $13 \%$ \\
Estados Unidos & $70 \%$ & $7 \%$ \\
França & $14 \%$ & $10 \%$ \\
Reino Unido & $41 \%$ & $9 \%$ \\
\hline
\end{tabular}

Tabela 8. Estudo comparativo: "Recruiters and HR professionals who have rejected candidates based on data found online vs. consumers who think online data affected their job search" - Cross-Tab (2010).

Fonte: Cross-Tab (2010, p. 5).

Notas: Estudo com a referencia "B" na Tabela 1.

A gestão dessa "identidade digital" pode revelar-se uma tarefa complexa, onde, de forma "inconsciente", o próprio lhe pode associar informação que lhe será prejudicial:

Muitas vezes desprovidos de uma consciência digital - consciência relativamente ao peso, relevância e repercussão da informação que disponibilizam na rede - os indivíduos associam à sua identidade online conteúdos e informações que, recuperados de forma descontextualizada, poderão estar na origem de interpretações e representações erróneas da sua própria identidade (Aresta, 2013, p. 37).

Associando a imagem pessoal que qualquer pessoa constrói, mesmo sem intenção, com a sua atividade em linha, à persistência dos dados digitais (Aresta, 2013, p. 36), a simples utilização das RSeLs pode transformar-se numa “tatuagem eletrónica”, usando a designação de Juan Enriquez (Enriquez, 2013, 1:05).

Talvez devido à perceção dessa persistência, profissionais ligados aos recursos humanos, recomendam um cuidado continuado na manutenção dessa "identidade digital":

A nossa presença no digital é, neste momento, obrigatória. Deve ser planeada de forma a mostrar consistência e deve ser "alimentada", ou seja, devemos saber criar um buzz. Creio que não vale a pena estar sempre a "falar", mas é importante expressarmos a nossa visão das coisas, mostrar um papel interventivo na sociedade e partilhar conhecimento. Sobretudo isso, partilhar conhecimento (Delgado, comunicação pessoal, maio 13, 2015). 
Segundo Berkelaar \& Buzzanell, alguns recrutadores chegam mesmo a esperar que os candidatos qualificados mantenham essa "online professional presence across time, space, and (role) contexts previously excluded from personnel selection” (Berkelaar \& Buzzanell, 2015, p. 106).

Por outro lado, a ausência de informação poderá ser também um fator negativo para os candidatos, pois os recrutadores também podem avaliar esse aspeto: "they also evaluate candidates based on absent information they expected to find” (Berkelaar \& Buzzanell, 2015, p. 103), até porque chega a funcionar como comprovativo das informações constantes no currículo tradicional impresso (Molina, 2011, p. 67).

\section{RESULTADOS E DISCUSSÃO}

Com este trabalho, foi possível obter uma visão abrangente sobre a importância, em termos profissionais, da atividade desenvolvida em linha, em particular nas "Redes Sociais". Dessa forma é possível responder às três questões formuladas:

a) Os responsáveis pelos recursos humanos e/ou outros com poder de decisão em termos de gestão de funcionários, procuram efetivamente informação em linha sobre potenciais funcionários?

- Sim, essa verificação (denominada de cybervetting) é efetuada ao longo do processo de seleção, embora não seja possível determinar com exatidão a real expressividade e frequência dessa prática.

b) A informação encontrada tem um papel relevante na decisão desses gestores de recursos humanos?

- Os dados consultados apontam para uma resposta afirmativa, das treze percentagens em termos de decisão influenciada pela informação obtida em linha, nove são iguais ou superiores a quarenta e nove por cento. Dessas nove, cinco dizem respeito a decisões prejudiciais aos candidatos e apenas uma a decisões positivas. As outras três, os estudos não as especificam pelo que poderão englobar ambos os tipos de decisão.

c) Quem procura trabalho deve cuidar da sua "imagem digital" mesmo nas RSeLs não consideradas profissionais?

- Tendo em conta que a "imagem pessoal digital”, ou "identidade digital”, é criada a partir de toda informação encontrada em linha, associada à pessoa em questão, todas as RSeLs têm relevância. Sendo, frequentemente, essa identidade digital a primeira imagem que os empregadores têm dos aspirantes às ofertas de emprego, descurar esse aspeto é uma atitude que pode levar os candidatos a serem excluídos do processo de recrutamento logo à entrada.

Na bibliografia consultada, é possível constatar que a preocupação pelas questões legais e éticas, levantadas pela prática de cybervetting, nomeadamente a possibilidade de invasão da privacidade, está presente no discurso dos recrutadores (L'Atelier, 2009; Openshaw, 2011, p. 5; Robert Walter Group, 2013, p. 10; SHRM, 2008b, p. 2) No entanto, entre o discurso e o comportamento descrito, uma certa falta de assertividade parece estar presente, seja por pressões relacionadas com o processo de recrutamento (Berkelaar \& Buzzanell, 2015, p. 108), seja por questões de salvaguarda pessoal e institucional, os recrutadores sentem a necessidade de efetuar as devidas diligências a fim de evitar surpresas (Bashford, 2008; Berkelaar e Buzzanell, 2014, p. 464; Lermusi, 2011). E, mesmo que os profissionais de recursos humanos se abstenham de usar a prática de cybervetting, devido a questões legais e éticas, nada garante que o responsável último pelo recrutamento não o faça (Lermusi, 2011; Pereira, 2013).

A maioria dos estudos apontam para um aumento da utilização, por parte de quem contrata, da pesquisa de informação publicada em linha, sobre os candidatos às ofertas de emprego (Berkelaar e Buzzanell, 2014, p. 456; Broughton et al., 2013, p. 35; Fernandes, 2014, p. 55; A. F. P. Gomes, 2011, p. 54; T. C. Gomes et al., 2012, p. 15; Jobvite, 2014, p. 2; Martins, 2014, p. 30; Molina, 2011, p. 80; SHRM, 2008b, p. 7; Vieira, 2012, p. 68). Notando-se, atendendo aos estudos consultados, nos recrutadores portugueses e brasileiros, uma tendência para recorrerem cada vez mais a esse expediente. Citando Teresa Calisto, profissional ligada aos recursos humanos, "a pesquisa é feita e o que encontram vai naturalmente moldar a sua opinião, juntamente com os outros elementos formais apresentados numa candidatura de emprego" (Calisto, comunicação pessoal, junho 12, 2015).

Paradoxalmente, quanto mais a atividade desenvolvida nas RSeLs se assemelha à realizada nas Redes Socias "fora da rede", maior consciencialização o indivíduo terá de ter das particularidades dos dois “espaços”. Como expõe Pedro Xavier Mendonça, investigador académico em estudos sociais de tecnologia e de comunicação, referindo-se ao paradigmático Facebook:

A relação entre os espaços privado e público alterou-se. O esforço que o Facebook faz para manter a aparência de privacidade, de lugar doméstico, entre amigos, cercado de afetos partilhados entre pares cúmplices e exclusivos é constantemente atravessado por aquilo que faz do Facebook o Facebook: é social. Nada se faz ali sem uma relação com o domínio público (Mendonça, 2013). 
Mendonça aborda a fusão entre o espaço privado e o espaço público nas RSeLs que será, em última análise, a grande responsável pelas questões legais e éticas levantadas com a prática de cybervetting. Embora não se tenha pretendido realizar uma análise desta problemática em termos éticos ou legais, o que aqui foi abordado vai ao encontro à opinião de Moreira (2013, p. 78), "we think that it is very difficult to the candidate, or even impossible, to prove that he/she was excluded of the hiring process by the research that the future employer made online". E, mesmo que essa prova seja efetuada, segundo Veríssimo, Macias, e Rodrigues (2012, p. 22) a tendência internacional da jurisprudência "é considerar as redes sociais como enquadradas na esfera pública do utilizador, independentemente das suas definições de privacidade".

\section{CONSIDERAÇÕES FINAIS}

O esbatimento entre o espaço privado e público torna relevante, em termos profissionais, toda a informação publicada em linha associada à nossa pessoa. Porque, como foi descrito no presente trabalho, é a imagem construída pelo recrutador (neste caso em particular), a partir do conjunto da informação recolhida em linha (independente do contexto ou assunto) associada a um indivíduo em particular, que é considerada como representativa do modo de ser da pessoa em questão.

É nesse sentido que o conceito "identidade digital" surge no presente trabalho, enquanto cópia digital das atitudes e comportamentos, da pessoa em questão, fora da rede. Ao contrário da "identidade online", associada apenas às manifestações em linha do indivíduo (Aresta, 2013, p. 27; Kokswijk, 2007, p. 83); a "identidade digital" é percebida como tendo uma ligação direta com a identidade fora de linha, a chamada "identidade física" ou "real". É esse entendimento, por parte de quem contrata, que faz da "atividade social" realizada em linha um fator de influência, por vezes decisiva, no processo de seleção e recrutamento.

Outras abordagens e metodologias seriam necessárias para um aprofundamento das relações entre estas várias "identidades" no sentido de aferir a legitimidade da inferência da personalidade de uma pessoa, a partir da informação recolhida no seu perfil nas RSeLs.

Este trabalho apresenta limitações inerentes ao seu cariz exploratório dado a problemática abordada ser um assunto ainda pouco aprofundado, em especial se a área geográfica de incidência dos estudos for limitada a Portugal e Brasil. Dos oito trabalhos consultados, cujo estudo diz respeito a esses dois países, cinco referem essa limitação em termos de fontes de referência, (Almeri et al., 2013, p. 91; Fernandes, 2014, p. 6; A. F. P. Gomes, 2011, p. 41; Martins, 2014, p. 3; Molina, 2011, p. 5) sendo que destes, dois do Brasil (Almeri et al., 2013; Molina, 2011) e um de Portugal (Martins, 2014), não restringem a sua afirmação ao respetivo país.

Dessa limitação nasceu a necessidade de incluir estudos cuja incidência abrangesse outras áreas geográficas. Para além de, só com esse alargamento, ter sido possível obter outras perspetivas sobre a matéria, para além da académica, como era a intenção do trabalho.

Um maior alargamento, aplicado de forma sistemática a bases de dados internacionais, seria necessário para um maior entendimento e aprofundamento desta problemática. Não só relativamente à real expressividade, frequência e relevância da prática de cybervetting, como também no sentido de abordar a perspetiva dos candidatos às ofertas de emprego e o feedback que os mesmos têm, se o têm, quando a decisão da sua não seleção foi baseada na informação encontrada nas RSeLs.

Concluindo, sobressai a premente necessidade de uma maior atenção ao que se publica em linha. Parafraseando uma afirmação do Blog: LIS 9763 Social Software and Libraries, "what happens online, stays online forever" (Reiterowski, 2013). Uma afirmação um pouco exagerada talvez, pois até as tatuagens se removem, é certo, mas não sem deixar sequelas. 


\section{REFERÊNCIAS}

Acquisti, A. (2013, 14 de Junho). What will a future without secrets look like? TED Conferences. Recuperado de http://www .ted.com/talks/alessandro_acquisti_why_privacy_matters

Almeri, T. M., Martins, K. R., Paula, D., \& Paiva, S. (2013). O uso das redes sociais virtuais nos processos de recrutamento e seleção. Educação, Cultura e Comunicação, 4(8), 77-94. Recuperado de http://publicacoes.fatea.br/index.php/eccom/ article/viewFile/635/453

Araújo, V., Cardoso, G., \& Espanha, R. (2008). Social structures in the era of networked communication: Youth and the reshaping of mediation. Lisboa: ISCTE - Lisbon Internet and Networks Institute. (LINI Working Papers N. 3)

Archana, L., Nivya, V. G., \& Thankam, S. M. (2008). Recruitment through social media, area: Human resource. IOSR Journal of Business and Management, 37-41. Recuperado de http://iosrjournals.org/iosr-jbm/papers/ncibppte-volume -1/1024.pdf

Aresta, M. S. L. (2013). A construção da identidade em ambientes digitais: estudo de caso sobre a construção da identidade online no Sapo Campus e em ambientes informais (Tese de Doutorado, Universidade de Aveiro). Recuperado de http://ria.ua.pt/handle/10773/10441

Bashford, S. (2008, 22 de Janeiro). Recruitment: Virtual vacancies. Recuperado em 18 jun. 2015, de http://www.marketingmagazine.co.uk/article/778589/ recruitment-virtual-vacancies

Berkelaar, B. L., \& Buzzanell, P. M. (2014). Cybervetting, person-environment fit, and personnel selection: Employers' surveillance and sensemaking of job applicants' online information. Journal of Applied Communication Research, 42(4), 456-476. doi: 10.1177/0893318914554657

Berkelaar, B. L., \& Buzzanell, P. M. (2015). Online employment screening and digital career capital: Exploring employers' use of online information for personnel selection. Management Communication Quarterly, 29(1), 84-113. doi: 10.1080/00909882.2014.954595

Broughton, A., Foley, B., Ledermaier, S., \& Cox, A. (2013). The use of social media in the recruitment process (no. 03/13). Brighton: ACAS - Advisory, Conciliation and Arbitration Service. Recuperado de http://www.acas.org.uk/media/pdf/0/b/ The-use-of-social-media-in-the-recruitment-process.pdf

Campos, M. D., \& Oliveira, H. (2013, Maio). Despedidos por causa de mensagens no facebook: Apanhados na rede. Visão(1054), 58-63. Recuperado de http://issuu.com/ medianasa/docs/vis_o__apanhados_na_rede/5

Cardoso, G., Araújo, V., \& Espanha, R. (2009). Out of information and into communication: Networked communication and internet usage. Lisboa: ISCTE - Lisbon Internet and Networks Institute. Recuperado de http://www.lini-research.org/np4/?newsId=11\&fileName= GCARDOSO_VARAUJO_LINI_WP5.pdf (LINI Working Papers N.5)

Clark, L. A., \& Roberts, S. J. (2010). Employer's use of social networking sites: A socially irresponsible practice. Journal of Business Ethics, 95(4), 507-525. doi: 10.1007/s10551-0100436-y

Council of Europe. (2015). Recommendation CM/Rec(2015) 5 of the Committee of Ministers to member States on the processing of personal data in the context of employment (2015). Recuperado de http://www.dgpj.mj.pt/sections/noticias/ conselho-da-europa_3/downloadFile/attachedFile_f0/

\section{CMRec20155E.pdf?nocache $=1428485746.25$}

Cross-Tab. (2010). Online reputation in a connected world. Cross-Tab Marketing Service. Recuperado de http://www.job-hunt.org/guides/DPD_Online-Reputation -Research_overview.pdf

Davison, H. K., Maraist, C. C., Hamilton, R. H., \& Bing, M. N. (2012). To screen or not to screen? using the internet for selection decisions. Employee Responsibilities and Rights Journal, 24(1), 1-21. doi: 10.1007/s10672-011-9178-y

Ellison, N. B., \& Boyd, D. M. (2013). Sociality through social network sites. In W. H. Dutton (Ed.), The oxford handbook of internet studies (p. 151-172). Oxford: Oxford University. doi: 10.1093/oxfordhb/9780199589074.001.0001

Enriquez, J. (2013, Fevereiro). Your online life, permanent as a tattoo. TED Conferences. Recuperado de http://www.ted.com/talks/juan_enriquez_how_to_think _about_digital_tattoos

Fernandes, M. C. F. (2014). O uso das redes sociais no recrutamento externo em empresas portuguesas. Recuperado de http://recipp.ipp.pt/handle/10400.22/5266 (Instituto Politécnico do Porto - Escola Superior de Estudos Industriais)

Gil, A. C. (2008). Métodos e técnicas de pesquisa social (6a. ed.). São Paulo: Atlas.

Gomes, A. F. P. (2011). Recrutamento nas redes sociais on-line. Lisboa: Instituto Superior de Economia e Gestão. Recuperado de https:/www.repository.utl.pt/handle/10400.5/3394

Gomes, T. C., Scherer, L. A., \& Lobler, M. L. (2012). Uso das redes sociais virtuais no processo de recrutamento e seleção de pessoal: Uma análise na perspectiva de profissionais de recursos humanos. In Simpoi (p. 1-17). São Paulo: Fundação Getúlio Vargas. Recuperado de http://www.simpoi.fgvsp.br/ arquivo/2012/artigos/E2012_T00264_PCN12758.pdf

Harvard Medical School. (2012, 17 de Fevereiro). Dawn of social networks: Hunter-gatherers provide clues about the evolution of cooperation. Recuperado de https://youtu.be/ It-pHyDrkTM

Jobvite. (2010). 2010: Social recruiting survey results. Recuperado de http://web.jobvite.com/rs/jobvite/images/ Jobvite2010SocialRecruitingReport_2.pdf

Jobvite. (2014). 2014: Social recruiting survey. Recuperado de https://www.jobvite.com/wp-content/uploads/2014/10/ Jobvite_SocialRecruiting_Survey2014.pdf

Kokswijk, J. V. (2007). Digital ego: Social and legal aspects of virtual identity. Delft: Eburon.

L’Atelier. (2009, 24 de Agosto). Ética e recrutamento via redes sociais. Recuperado de http://www.e-thesis.inf.br/index.php ?option=com_content \&task=view \&id=5783 \&Itemid=142

Lermusi, Y. (2011, 14 de Setembro). Cyber-vetting's usage, risk, and future. Recuperado em 4 July 2015, de http://www .eremedia.com/ere/cyber-vettings-usage-risk-and-future/

Macleod, I. (2011, 24 de Outubro). 91\% of employers use social media to screen applicants. Recuperado em 26 jun. 2015, de http://www.thedrum.com/news/2011/10/24/ 91-employers-use-social-media-screen-applicants

Martins, S. F. L. (2014). A procura online dos talentos: o papel dos sites de redes sociais no recrutamento de candidatos (Dissertação de Mestrado, Universidade de Lisboa - Instituto Superior de Ciências Sociais e Políticas, Lisboa). Recuperado de http://hdl.handle.net/10400.5/8324

Mendonça, P. X. (2013, 27 de Dezembro). O paradoxo do Facebook: Despedimentos e direito ao topless. Recupe- 
rado de http://expresso.sapo.pt/blogues/bloguet_ciencia/ blogue na casa das maquinas/o-paradoxo-do-facebook -despedimentos-e-direito-ao-topless $=\mathrm{f} 847899$

Molina, M. V. L. F. (2011). Redes sociais virtuais como uma nova ferramenta nos processos de recrutamento, seleção e controle de pessoal (Monografia de Graduação, Universidade de Brasília - Faculdade de Economia, Administração e Contabilidade, Brasília). Recuperado de http://bdm.unb.br/handle/ $10483 / 4231$

Moreira, T. C. (2013). The digital to be or not to be: Privacy of employees and the use of online social networks in hiring decisions. International Journal of Law and Social Sciences, 2(2). Recuperado de http://dl6.globalstf.org/index.php/jlss/ article/viewFile/531/548

Openshaw, A. (2011). The impact of social media on recruitment. Recuperado de http://www.nigelwright.com/media/ 1032/the-impact-of-social-media-on-recruitment.pdf

Pereira, J. P. (2013, 29 de Abril). Se procura emprego, não aposte tudo na internet. Recuperado de http://www.publico.pt/tecnologia/noticia/se-procura -emprego-nao-aposte-tudo-na-internet-1592854

Real, M. C., Cardoso, M. S., Giovaneta, A., \& Birrer, B. (2012). A influência das redes sociais virtuais no processo de recrutamento e seleção das empresas associadas à AJESM. In 1o. fórum internacional ecoinovar. Santa Maria: Universidade Federal de Santa Maria. Recuperado de http://ecoinovar.com.br/ cd/artigos/ECO040.pdf

Reiterowski, A. (2013, 21 de Fevereiro). Social media policies and assessment. Recuperado em 23 June 2015, de https://alyreiterowski.wordpress.com/2013/02/21/social -media-policies-and-assessment/

Robert Walter Group. (2013). Using social media in the recruitment process. London. Recuperado de http://www.robertwalters.co.uk/wwwmedialibrary/ WWW2/country/united-kingdom/content/Whitepaper/ using-social-media-in-the-recruitment-process.pdf (Robert Walters Whitepaper)

Saramago, J. (2015, 25 de Abril). Europa proíbe despedimentos. Correio da Manhã. Recuperado de http://www.cmjornal.xl.pt/nacional/detalhe/ europa_proibe_despedimentos.html

SIC Notícias. (2015, 24 de Abril). Conselho da Europa proíbe patrões de vigiar empregados nas redes sociais. Recuperado de http://sicnoticias.sapo.pt/pais/ 2015-04-24-Conselho-da-Europa-proibe-patroes-de-vigiar -empregados-nas-redes-sociais

Society for Human Resource Management. (2008a). Online technologies and their impact on recruitment strategies: Using social networking websites to attract talent. Recuperado de http://www.shrm.org/research/surveyfindings/ documents/SNSstaffingresearchPresentationFinal.pdf

Society for Human Resource Management. (2008b). SHRM staffing research: Online technologies and their impact on recruitment strategies. Recuperado de http://www.shrm.org/Research/SurveyFindings/ Documents/08-0521StaffInsert_3rdQtr_FINAL.pdf

Society for Human Resource Management.

(2011). SHRM survey findings: The use of social networking websites and online search engines in screening job candidates. Recuperado de http://www.shrm.org/Research/ SurveyFindings/Documents/Social\%20Networking\% 20Websites\%20Recruiting\%20and\%20Staffing_FINAL.pptx

Veríssimo, J., Macias, M., \& Rodrigues, S. (2012). O im- pacto jurídico das redes sociais na internet (Dissertação de Mestrado, Universidade Nova de Lisboa - Faculdade de Direito). Recuperado de http://www.fd.unl.pt/docentes_docs/ ma/meg_ma_15739.pdf

Vieira, M. P. S. (2012). Impacto das novas tecnologias no recrutamento nas empresas especializadas de recrutamento e selecção (Dissertação de Mestrado, Instituto Universitário de Lisboa - ISCTE Business School). Recuperado de https:// repositorio.iscte-iul.pt/handle/10071/4031

WETM-TV. (2014, 8 de Abril). Corning Community College students learn about social media impacts. Recuperado de http://www.mytwintiers.com/story/d/story/ corning-community-college-students-learn-about-soc/ 35451/isMc1z1OokOpgoxLfz4UxA
Como citar este artigo (APA):

Machado, L. M. O. (2016). A influência da informação publicada nas "redes sociais" no processo de seleção e recrutamento: um estudo exploratório na literatura. AtoZ: novas práticas em informação e conhecimento, 5(1), 10 - 20. Recuperado de: http://dx.doi.org/ 10.5380/atoz.v5i1.44035 